\title{
A population approach to characterise amisulpride pharmacokinetics in older people and Alzheimer's disease
}

\author{
Suzanne Reeves ${ }^{1,2}$ • Julie Bertrand ${ }^{3}$ • Fabrizia D'Antonio ${ }^{1,2}$ • Emma McLachlan ${ }^{2}$. \\ Akshay Nair ${ }^{1} \cdot$ Stuart Brownings ${ }^{2} \cdot$ Suki Greaves $^{4} \cdot$ Alan Smith $^{4}$ • David Taylor ${ }^{4}$. \\ Robert Howard ${ }^{1,2}$
}

Received: 14 April 2016 / Accepted: 30 June 2016 /Published online: 1 August 2016

(C) The Author(s) 2016. This article is published with open access at Springerlink.com

\begin{abstract}
Introduction Current prescribing guidelines for the antipsychotic amisulpride are based largely on pharmacokinetic (PK) studies in young adults, and there is a relative absence of data on older patients, who are at greatest risk of developing adverse events. Methods This study aimed to develop a population PK model for amisulpride specifically in older people, by combining data from a richly sampled phase 1 , single $(50 \mathrm{mg})$ dose study in healthy older people ( $n=20,65-79$ years), with a clinical dataset obtained during off label, low-dose (25-75 mg daily) amisulpride prescribing in older people with Alzheimer's disease (AD) $(n=25,69$ 92 years), as part of an observational study.

Results After introducing a scaling factor based on body weight, age accounted for $20 \%$ of the inter-individual variability in drug clearance (CL), resulting in a $54 \%$ difference in CL between those aged 65 and those aged 85 years, and higher blood concentrations in older patients.

Discussion These findings argue for the consideration of age and weight-based dose stratification to optimise amisulpride prescribing in older people, particularly in those aged 85 years and above.
\end{abstract}

Electronic supplementary material The online version of this article (doi:10.1007/s00213-016-4379-6) contains supplementary material, which is available to authorized users.

Suzanne Reeves

suzanne.reeves@ucl.ac.uk

1 Division of Psychiatry, University College London, London W1T7NF, UK

2 Department of Old Age Psychiatry, Kings College London, London, UK

3 UMR 1137 IAME INSERM University Paris 7, France and Genetics Institute, University College London, London, UK

4 South London and Maudsley NHS Foundation Trust, London, UK
Keywords Amisulpride · Elderly · Alzheimer's disease · Age $\cdot$ Population pharmacokinetics $\cdot$ Antipsychotic

\section{Introduction}

Safe and effective prescribing of antipsychotic medication is challenging in older people, as they are extremely susceptible to adverse events, including extrapyramidal side effects (EPS), falls, sedation and postural hypotension (Jeste et al. 2008; Leon et al. 2010). The mechanisms underpinning this heightened sensitivity are poorly understood (Uchida et al. 2009b), and research which aims to establish pharmacokinetic (PK) and pharmacodynamic (PD) contributions to clinical response and side effects in older clinical populations will be a key step towards improving safety profiles (Bigos et al. 2008a; Lotrich et al. 2006). This issue is particularly pertinent for older people with dementia, in whom excessive morbidity and increased cerebrovascular mortality have led to a restriction of antipsychotic use, and an emphasis on safety monitoring, but no clear guidance on minimum clinically effective doses when antipsychotic drugs are prescribed off licence to treat psychotic symptoms (Jennum et al. 2015; Maust et al. 2015).

The most widely held assumption is that age-related changes in peripheral pharmacokinetics, including changes in body composition, and a reduction in hepatic metabolism and renal clearance, lead to higher blood concentrations for a given drug dose (Mangoni and Jackson 2004; Merle et al. 2005). However, studies that have used a population approach (Duffull et al. 2011; Ette et al. 2004) to investigate PK profiles of antipsychotic drugs in older patients have shown that an age effect on drug clearance is not generalizable across antipsychotics (Bigos et al. 2008b; Feng et al. 2008), and emphasise the need to develop PK models for individual drugs.

Amisulpride is a second-generation antipsychotic drug widely used for the treatment of schizophrenia (Mauri et al. 2014), and 
for which optimal dose (400-800 mg daily), and therapeutic range of dopamine (D2/3) receptor occupancy (40-70\%), and blood concentration (100-319 $\mathrm{ng} / \mathrm{ml})$ have been clearly established (Hiemke et al. 2011; Lako et al. 2013; Sparshatt et al. 2009). These recommendations are based largely on patients below the age of 65 years, with minimal data on those aged 80 years and above. Pharmacokinetic studies of amisulpride (Coukell and Benfield 1996; Hamon-Vilcot et al. 1998; Rosenzweig et al. 2002) describe rapid absorption following oral administration, achieving peak plasma concentration (Cmax) after $1 \mathrm{~h}$, and a second peak after $3 \mathrm{~h}$, consistent with hepatobiliary elimination. The drug has low bioavailability (48 \%) and low plasma protein binding (17\%) and is not a cytochrome P450 substrate. Amisulpride is eliminated unchanged in the urine (elimination half-life $\left(t^{1 / 2}\right) 12 \mathrm{~h}$ ) and has high renal clearance (330 ml/min), suggestive of additional renal secretion (Dufour and Desanti 1988), possibly via the organic cation transport (OCT) system (Dos Santos Pereira et al. 2014; Jonker and Schinkel 2004). PK data on healthy older people are limited to a phase 1 study, which examined amisulpride PK characteristics in the first $72 \mathrm{~h}$ following a single $(50 \mathrm{mg})$ dose (Hamon-Vilcot et al. 1998).

We have recently collected PK, $\left[{ }^{18} \mathrm{~F}\right]$ fallypride $\mathrm{D} 2 / 3$ receptor imaging and clinical outcome data in older patients with $\mathrm{AD}$ who were prescribed amisulpride (25-75 mg daily) off label to treat psychotic symptoms, as part of an open observational study. To maximise the potential utility of this dataset to inform safer prescribing in $\mathrm{AD}$, we aimed to use a population approach to establish the consistency and identify sources of variability in PK-PD relationships. This analysis represents the first stage of model development, with the following aims:

1. To develop a population PK model for amisulpride specifically for older people and $\mathrm{AD}$, by combining the clinical dataset with published data from the single $(50 \mathrm{mg})$ dose study

2. To investigate the contribution of physiological characteristics to inter-individual variability in PK parameters

3. To use model outputs to simulate and predict amisulpride dose-concentration relationships in people aged 65 years and over.

\section{Methods}

\section{Data sources}

\section{Group 1}

Twenty healthy elderly participants participated in a two-centre open study, which was approved by the Ethics Committee of the Pitie Salpetriere Hospital (Paris) (Hamon-Vilcot et al. 1998).
Participants were included on the basis of having no haematological or biochemical abnormalities and were on no concomitant medication. Verbal and written informed consent was obtained prior to inclusion. Participants were sampled before and at $1,2,3,4,5,6,8,10,12,24,32,48$ and $72 \mathrm{~h}$ following a single tablet of $50 \mathrm{mg}$ amisulpride, administered in the morning. Bloods concentrations of the amisulpride racemate were measured using a validated HPLC method based on liquid-liquid extraction and fluorescence detection. The method is linear from $0.5 \pm 640 \mathrm{ng} / \mathrm{ml}$ in plasma, with a limit of quantification of $0.5 \mathrm{ng} / \mathrm{ml}$. Both the racemate and enantiomers of amisulpride were measured, but only data on the racemate was used in the current analysis, for consistency with group 2 data.

\section{Group 2}

PK data were obtained from 25 patients with a diagnosis of probable AD (McKhann et al. 1984), who were participating in an observational study of amisulpride prescribing in older people. Patients were recruited from older adult mental health services based within the catchment area of the South London and Maudsley NHS Foundation Trust (SLaM) (London, UK), immediately prior to commencing low-dose amisulpride, which was being used off label to treat psychotic symptoms. Exclusion criteria included current or past history of psychiatric illness, being prescribed an antipsychotic or other oral drug that interferes with brain dopamine function, parkinsonian or other features suggestive of Lewy Body Dementia (McKeith et al. 1996), significant cardiorespiratory disease or needle phobia, and any contraindication to amisulpride use as stated in the summary of product characteristics ( $\mathrm{SmPc}$ ). Verbal and written informed consent was obtained from participants, or from a carer where a participant lacked capacity to give fully informed consent. The study was approved by the Berkshire Research Ethics Committee (REC reference 11/SC/0486). Clinical assessment was carried out at baseline and during dose titration: Psychotic symptoms and associated agitation were rated using the summed total score on three domains (delusions, hallucinations and agitation) of the carer-rated Neuropsychiatric Inventory (NPI) (Cummings et al. 1994), and extrapyramidal symptoms (EPS) were rated using the Simpson-Angus Scale (SAS) (Simpson and Angus 1970). Where possible, $\left[{ }^{18} \mathrm{~F}\right]$ fallypride dopamine D2/3 receptor positron emission tomography (PET) imaging was carried out prior to amisulpride being commenced and when an optimum dose was achieved (25\% reduction in symptoms). Patients commenced amisulpride at a dose of 25 or $50 \mathrm{mg}$ (based on the preference of the prescribing clinician), which was administered as a single evening dose, and increased to an optimum dose ( $25 \%$ or greater reduction in symptoms and minimal EPS). Flexibility was built into the design around the timing of followup assessments, to account for variability in the dose titration regimen across prescribers and ensure that amisulpride concentration was obtained prior to each dose increase. As samples were 
taken at least 1 week after commencing amisulpride, all samples were assumed to be at steady state. In compliance with medication (pill counts and discussion with carer), changes in concomitant medication and clinical outcome (symptom ratings, side effects) were recorded at each visit. The timing of blood collection was not controlled and reflected convenience samples, which coincided with follow-up assessments and/or imaging. Date, time of sample and hours since last dose (confirmed by a carer where possible) were recorded on the anonymised assay request form. Blood samples were analysed in a secure, CPAaccredited laboratory (Clinical Toxicology Unit, Kings College Hospital). Amisulpride (racemate) blood concentrations were determined using validated liquid chromatography with tandem mass spectrometry (LC-MS/MS) method, with a detection limit of $9 \mathrm{ng} / \mathrm{ml}$.

\section{Statistical analysis}

Data were analysed using SPSS (version 22.0). Gender differences in demographic and physiological characteristics were explored using independent samples $t$ tests. Correlations were expressed as Pearson's correlation coefficient $r$.

\section{Population PK analysis}

Nonlinear mixed effects modelling (NLME) was implemented using Monolix software (version 4.33; www.lixoft.eu). Diagnostic graphics and tests for covariate screening were performed in R. (version 3.2.4). Parameters were estimated using the Stochastic Approximation Expectation Minimisation (SAEM) algorithm (Kuhn and Lavielle 2005).

\section{Structural PK model}

An oral two-compartment model was developed for amisulpride, with five parameters: (i) an absorption constant (ka), (ii) a central compartment (V1), representing blood and well-perfused tissues (e.g. liver, kidney), (iii) a peripheral compartment (V2) representing less well-perfused tissues (e.g. muscle, lean tissue, fat), (iv) an inter-compartmental distribution constant (Q), and (v) an elimination constant (CL), which includes renal and systemic clearance. The PK model was initially described using data from the single dose study (group 1), with initial estimates for the parameters being guided by the literature (Coukell and Benfield 1996; HamonVilcot et al. 1998; Rosenzweig et al. 2002). Group 1 data was then combined with steady state data from AD patients (group 2). Blood concentration was converted from nanograms per millilitre to milligrams per litre for use in PK model building and concentrations below the limit of quantification (LOQ $5^{\mathrm{e}-4} \mathrm{mg} / 1$ for group 1 and $9^{\mathrm{e}-3} \mathrm{mg} / 1$ for group 2) accounted for through simulation of the censored data with a truncated Gaussian distribution in the SAEM-Markov Chain
Monte Carlo procedure (Samson and Lavielle 2006). Interindividual variability (IIV) for PK parameters was estimated using an exponential model $P_{i}=P_{T V} \times e^{\eta p i}$ where $P_{i}$ and $\eta_{p i}$ are the parameter estimate and corresponding random effect for the $i$ th individual, and $P_{T V}$ is the typical value for the parameter at the population level. The variability between the $i$ th individual and population parameter values was described by $\eta_{p i}$, which was assumed to be normally distributed with a mean of 0 and a variance of $\omega_{p}^{2}$. In addition to IIV, a proportional residual error model $\left(y_{i j}=\hat{y}_{i j}\left(1+\varepsilon_{i j}\right)\right)$ was used to describe random unexplained variability (system noise, dosage history errors and/or model misspecifications), where $y_{i j}$ and $\hat{y}_{i j}$ represent the $j$ th observed amisulpride concentration of the $i$ th subject, and its corresponding model predicted concentration, and $\varepsilon_{i j}$ was assumed to be normally distributed with a mean of 0 and a variance of $\sigma^{2}$. Residual error was modelled separately for each group, to account for inter-study differences.

\section{Covariate model}

The contribution of physiological characteristics to IIV was assessed in a covariate screening which included weight, height, age and gender, and creatinine clearance (CrCL), which was estimated using the Cockcroft and Gault equation (Cockcroft and Gault 1976) and converted to 1/h. Given the co-linearity between CrCL and other covariates of interest (weight, gender, age), serum creatinine was included as a separate covariate and allometric scaling for weight was introduced as an initial step. For group 1, clearance parameters $(\mathrm{CL}, \mathrm{Q})$ were set proportional to the body weight ratio over $70 \mathrm{~kg}$ to the power 0.75 , and on the volume parameters (V1, $\mathrm{V} 2$ ) proportional to the body weight ratio over $70 \mathrm{~kg}$ to the power 1 (Mould et al. 2002). Covariates were then added in a stepwise procedure after visual inspection of covariate plots (continuous covariates were excluded if the correlation coefficient $r$ between covariate and parameter was $<0.25$ ) and regression analysis in $\mathrm{R}$. Continuous covariates which passed initial screening were introduced into the model after log transforming and centering on the mean. Gender was included as a categorical covariate. For the combined dataset, the absence of data on early time points meant that it was necessary to restrict allometric scaling for weight, and subsequent covariate testing, to CL.

\section{Model evaluation}

Appropriateness of structural and covariate models was evaluated using goodness-of-fit criteria including diagnostic scatter plots, visual predictive checks, degree of shrinkage, change in IIV, model precision and likelihood ratio tests, with a $5 \%$ threshold. 


\section{Model predictions}

Population estimates for CL $\left(\mathrm{CL}_{\mathrm{TV}}\right)$ and effect size of covariates (age and weight) on CL were used to estimate CL in an individual $\left(\mathrm{CL}_{i}\right)$, for a given age and weight, using the equation:

$\mathrm{CL}_{\mathrm{i}}=\mathrm{CL}_{T V} *\left(\right.$ age $_{i} /$ mean age $^{\beta}{ }_{\text {age }, \mathrm{CL}} \times\left(\text { weight }_{i} / 70\right)^{0.75}$

Terminal half-life $\left(t^{1} / 2\right)$ was calculated as the ratio of $\log (2)$ and the second slope of elimination in the two-compartment model, itself derived from clearances and volumes population estimates.

\section{Model simulations}

One hundred people were simulated for amisulpride concentration $15 \mathrm{~h}$ post-dose in each of the following categories: 65 , 75 or 85 years; and of standard $(70 \mathrm{~kg})$ or low $(50 \mathrm{~kg})$ body weight, across the prescribed dose range $(25,50,75 \mathrm{mg}$ daily).

\section{Results}

\section{Sample characteristics}

\section{Group 1}

A total of 280 samples (14 per person) were obtained from 20 healthy elderly participants $(10$ men, mean age $=68.7 \pm 4.1)$, over $72 \mathrm{~h}$ following a single oral 50-mg dose of amisulpride. Physiological characteristics of the sample are described in Table 1. Weight $(p<0.01)$, height $(p<0.01)$ and serum creatinine $(p<0.05)$ were significantly higher in men. CrCL was correlated with weight $(r=0.53, p<0.05)$ and creatinine $(r=-0.52, p<0.05)$ but not age $(r=-0.32, p=0.16)$. A scatterplot of observed amisulpride blood concentration (mg/ 1) versus time (hours) since dose is shown in Fig. 1a. Cmax $(64.0 \pm 29.9 \mathrm{ng} / \mathrm{ml})$ was observed after $2.1 \pm 0.78 \mathrm{~h}$. Cmax was higher in women $(77.5 \pm 28.8 \mathrm{ng} / \mathrm{ml})$ than men $(50.5 \pm 25.4 \mathrm{ng} / \mathrm{ml})(p<0.05)$, but not correlated with age $(r=-0.11, p=0.64)$, height $(r=-0.33, p=0.15)$, weight $(r=-0.33, p=0.15)$ or $\operatorname{CrCL}(r=-0.17, p=0.48)$.

\section{Group 2}

A total of 41 samples (mean $=2 \pm 0.78)$ were taken from 25 patients with $\mathrm{AD}(8(32 \%)$ male; mean age $=82 \pm 6.6$ years mean Mini Mental State Examination $(\mathrm{MMSE})=18.4 \pm 5.4$ ) (Folstein et al. 1975), at 16.2 \pm 3.1 , over a dose range of 25 $75 \mathrm{mg}$ daily. All samples were taken at steady state and after $56.9 \pm 58$-day treatment. Physiological and clinical characteristics of AD patients are described in Table 2. Height $(p<0.05)$ and serum creatinine $(p<0.05)$ were higher in men. CrCL was significantly correlated with age $(r=-0.65$, $p<0.01)$, weight $(r=0.65, p<0.01)$ and serum creatinine $(r=-0.57, p<0.01)$. A scatterplot showing observed amisulpride concentration (mg/l) versus time (h) since dose is shown in Fig. 1b. There was wide ( $>10$-fold) variability in dose-corrected concentration (mean $=0.85 \pm 0.53 \mathrm{ng} / \mathrm{ml}$ ), which was correlated with age $(r=0.38, p=0.01)$, but not weight $(r=-0.04, p=0.81)$, or $\operatorname{CrCL}(r=0.05, p=0.77)$, with no gender differences $(p=0.88)$.

\section{PK model}

\section{Group 1}

An oral two-compartment model (parameterised in ka, CL, V1, Q, V2), which incorporated significant correlations for
Table 1 Physiological characteristics: group 1 (healthy elderly, single $50 \mathrm{mg}$ dose)

\begin{tabular}{llll}
\hline Variable & $\begin{array}{l}\text { Mean } \pm \text { SD } \\
\text { Total sample }(n=20)\end{array}$ & $\begin{array}{l}\text { Mean } \pm \text { SD } \\
\text { Men }(n=10)\end{array}$ & $\begin{array}{l}\text { Mean } \pm \text { SD } \\
\text { Women }(n=10)\end{array}$ \\
\hline Age (years) & $68.7 \pm 4.1$ & $70.4 \pm 5.1$ & $67 \pm 1.3$ \\
CrCL $(\mathrm{ml} / \mathrm{min})$ & $80.5 \pm 17.5$ & $86.3 \pm 18.2$ & $74.4 \pm 15.3$ \\
Weight $(\mathrm{kg})$ & $66.6 \pm 9.1$ & $73.7 \pm 5.3 * *$ & $59.5 \pm 6.1$ \\
BMI $\left(\mathrm{kg} / \mathrm{m}^{2}\right)$ & $24.1 \pm 2.4$ & $25.2 \pm 1.8^{*}$ & $22.9 \pm 2.5$ \\
Height $(\mathrm{m})$ & $1.7 \pm 0.1$ & $1.7 \pm 0.5 * *$ & $1.61 \pm 0.5$ \\
Creatinine $(\mu \mathrm{mol} / \mathrm{l})$ & $56 \pm 10.2$ & $61.1 \pm 9.7 *$ & $51.0 \pm 8.4$ \\
Cmax $(\mathrm{ng} / \mathrm{ml})$ & $64.0 \pm 29.9$ & $50.5 \pm 25.4^{*}$ & $77.5 \pm 28.8$ \\
Tmax (hours) & $2.1 \pm 0.8$ & $2.4 \pm 0.7$ & $1.8 \pm 0.8$ \\
\hline
\end{tabular}

Values for men and women were compared using independent samples $t$ tests)

CrCL estimated creatinine clearance ( $\mathrm{ml} / \mathrm{min}$ - converted to $1 / \mathrm{h}$ for the purposes of model building), $B M I$ body mass index $\left(\mathrm{kg} / \mathrm{m}^{2}\right)$, Cmax peak plasma concentration $(\mathrm{ng} / \mathrm{ml}$ - converted to $\mathrm{mg} / \mathrm{l}$ for the purposes of model building), Tmax time taken to achieve Cmax (hours)

${ }^{*} p<0.05 ; *{ }^{*} p<0.01$ 


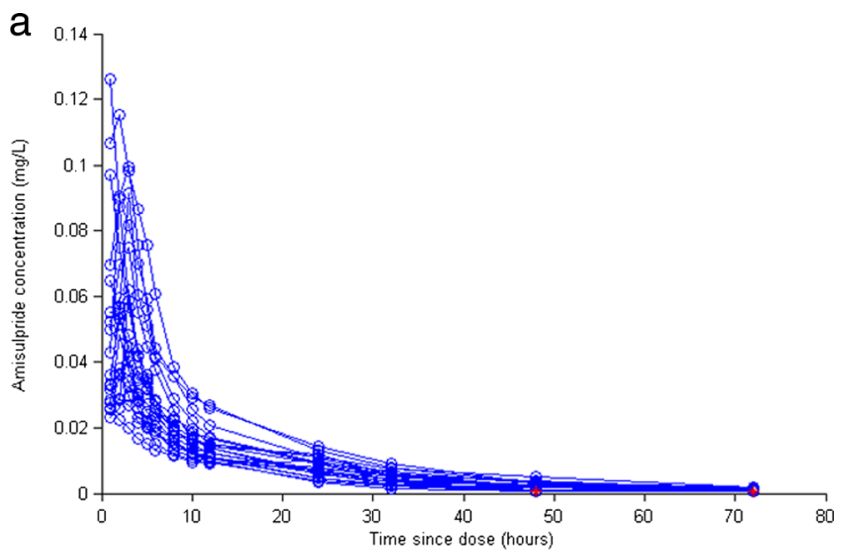

b

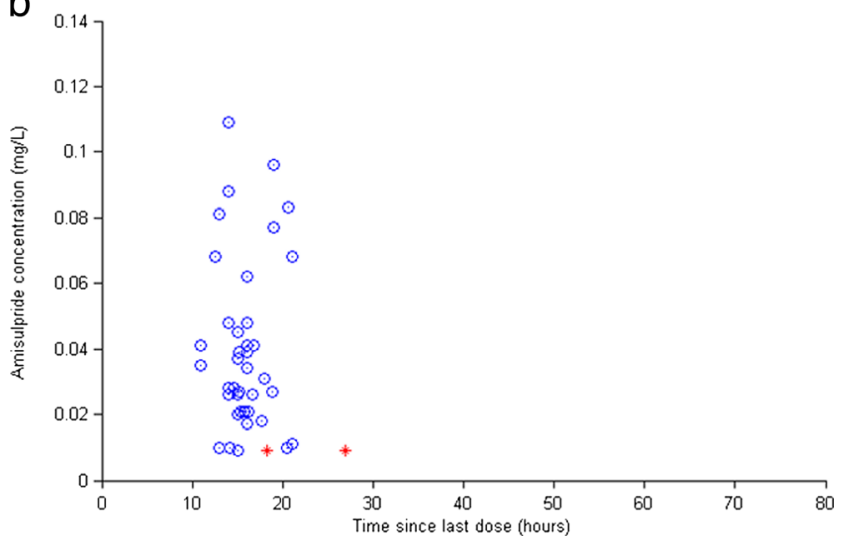

Fig. 1 Scatterplot showing time since dose versus observed amisulpride concentration (mg/l) in group 1 , following a single 50-mg oral dose (a), and group 2, at steady state across a dose range of 25-75 $\mathrm{mg}$ daily (b). Concentrations below the limit of the assay sensitivity are shown in red (colour figure online)

random effects between $\mathrm{CL}, \mathrm{Q}, \mathrm{V} 1$ and V2, was found to be the best fit for the data (see supplementary text and figures ESM 1, ESM 2). The model showed excellent precision, low $(<5 \%)$ shrinkage on clearance and volume parameters, and low $(13 \%)$ residual unexplained variability. Gender alone was a significant predictor of V1 $\left(\beta \_\mathrm{V} 1\right.$, gender $=-0.52, p=6.6^{\mathrm{e}-}$ $\left.{ }^{005}\right)$. Parameter estimates for the final model are summarised in Table 3, and visual predictive checks (VPC) shown in Fig. 2a. Population estimates for V1 were $40 \%$ lower in women $(399 \mathrm{l} / \mathrm{h})$ than men $(668 \mathrm{l} / \mathrm{h})$, and estimated $t^{1} / 2$ (based on a $70 \mathrm{~kg}$ person) was $13.6 \mathrm{~h}$ for women and $15.2 \mathrm{~h}$ for men.

\section{Combined dataset}

In the combined dataset, the population estimate for CL $(54.3 \mathrm{l} / \mathrm{h})$ was lower than that described for group 1 alone $(84 \mathrm{l} / \mathrm{h})$, and IIV on CL was higher $(56 \%$ in the combined, compared to $33 \%$ in the group 1 dataset). Residual unexplained variability (RUV) was also higher for group $2(53 \%)$ than group 1 (13\%), and shrinkage was $>45 \%$ for all parameters apart from CL $(27 \%)$. Age was identified as the only significant contributor to IIV in CL $\left(\beta C \mathrm{CL}\right.$, age $=-2.9, p=2.3^{\mathrm{e}-}$ ${ }^{7}$ ). Parameter estimates for the best fit model are shown in Table 3, and VPCs are shown separately for group 1 (Fig. 2b) and group 2 (Fig. 2c). Based on population PK parameters (in a person of standard $70 \mathrm{~kg}$ weight), the impact of age was such that CL in those aged 85 years $(41.8 \mathrm{l} / \mathrm{h})$ was $54 \%$ lower than those aged 65 years $(91.1 \mathrm{l} / \mathrm{h})$, and $t^{1 / 2}$ extended from 12.4 to $22.7 \mathrm{~h}$. Model simulations for amisulpride concentrations $15 \mathrm{~h}$ post-dose, accounting for age and body weight, are shown in Fig. 3.

\section{Discussion}

We have developed a population PK model for amisulpride in the elderly by combining data on healthy older people with a representative sample of AD patients, who were receiving low-dose amisulpride off label specifically to treat psychotic symptoms. The fact that PK data in group 1 was collected at multiple time points meant that it was possible to fully parameterise an oral two-compartment PK model and carry out covariate testing on all clearance and volume parameters. The final model explained $87 \%$ of the variance in PK profile with excellent precision and identified gender as a significant contributor to the variability in central volume of distribution (V1). Gender differences in V1, which led to a higher Cmax in women and longer estimated $t^{1} \frac{1}{2}$ in men $(15.3 \mathrm{~h}$ compared to $13.6 \mathrm{~h}$ in women), were not explained by differences in body mass, as weight was accounted for in the model through the use of allometric scaling, and height was compared against gender as part of covariate screening and model development.

The addition of group 2 data increased the estimated inter-individual variability in drug clearance from $31 \%$ (group 1 data alone) to $56 \%$ (combined sample). This variability was accounted for by a significant effect of age on drug clearance, consistent with but not solely accounted for an age-related reduction in CrCL. The magnitude of the effect was such that $\mathrm{CL}$ at 85 years was $54 \%$ lower than at 65 years, resulting in increased concentrations for a given dose in older patients. Whether the observed age effect represents primarily an effect of age on excretion, secretion or possibly both is unclear, but this warrants further investigation, as it has implications for the prescribing of other OCT substrates, such as clozapine (Haenisch et al. 2012), in older patients. It will also be important to further explore the nature of the age effect on CL, as it is likely that the effect of age increases exponentially across the 
Table 2 Sample characteristics: group 2 (Alzheimer's disease, steady state amisulpride treatment)

\begin{tabular}{llll}
\hline Variable & $\begin{array}{l}\text { Mean } \pm \text { SD } \\
\text { Total sample }(n=25)\end{array}$ & $\begin{array}{l}\text { Mean } \pm \text { SD } \\
\text { Men }(n=9)\end{array}$ & $\begin{array}{l}\text { Mean } \pm \text { SD } \\
\text { Women }(n=16)\end{array}$ \\
\hline Age (years) & $81.8 \pm 6.6$ & $81.8 \pm 7.7$ & $81.8 \pm 6.1$ \\
MMSE (maximum 30) & $18.4 \pm 5.4$ & $19.8 \pm 4.3$ & $17.7 \pm 5.8$ \\
CrCL (ml/min) & $67.7 \pm 17.3$ & $67.8 \pm 20.9$ & $67.7 \pm 17.4$ \\
Weight $(\mathrm{kg})$ & $68.0 \pm 15.2$ & $72.0 \pm 8$ & $65.8 \pm 18$ \\
BMI $\left(\mathrm{kg} / \mathrm{m}^{2}\right)$ & $26.5 \pm 5.4$ & $25.8 \pm 1.8$ & $26.9 \pm 6.7$ \\
Height $(\mathrm{m})$ & $1.6 \pm 0.1$ & $1.7 \pm 0.1 *$ & $1.6 \pm 0.1$ \\
Creatinine $(\mu \mathrm{mol} / \mathrm{l})$ & $83.1 \pm 25.7$ & $97.4 \pm 31.3 *$ & $75.1 \pm 18.5$ \\
Daily dose at time of sampling $(\mathrm{mg})$ & $49.4 \pm 11.2$ & $48.3 \pm 6.4$ & $48.1 \pm 13.7$ \\
Time since dose $(\mathrm{h})$ & $16.2 \pm 3.1$ & $15.8 \pm 3.2$ & $16.3 \pm 2.9$ \\
Days treatment, current dose & $56.9 \pm 58$ & $62.6 \pm 68.8$ & $57.4 \pm 56.8$ \\
Amisulpride concentration $(\mathrm{ng} / \mathrm{ml})$ & $40.9 \pm 27.1$ & $41.5 \pm 26.2$ & $40.5 \pm 28.1$ \\
Dose-corrected concentration $(\mathrm{ng} / \mathrm{ml} / \mathrm{mg})$ & $0.85 \pm 0.53$ & $0.87 \pm 0.52$ & $0.84 \pm 0.54$ \\
\hline
\end{tabular}

Values for men and women were compared using independent samples $t$ tests

MMSE Mini Mental State Examination, $C r C L$ estimated creatinine clearance $(\mathrm{ml} / \mathrm{min}$ - converted to $1 / \mathrm{h}$ for the purposes of model building, $B M I$ body mass index $\left(\mathrm{kg} / \mathrm{m}^{2}\right), C$ max peak plasma concentration $(\mathrm{ng} / \mathrm{ml}$ - converted to $1 / \mathrm{h}$ for the purposes of model building

${ }^{*} p<0.05$ age spectrum, resulting in changes in $\mathrm{CL}$ of a higher order of magnitude in the oldest individuals.

Introducing allometric scaling into the covariate model (Holford 1996; Mould et al. 2002) allowed CL to be estimated across the wide range of observed weights in the clinical dataset. Model simulations suggest that steady state blood concentrations at $50 \mathrm{mg}$ amisulpride daily would increase from 30 to $85 \mathrm{ng} / \mathrm{ml}$ between the ages of 65 and 85 years in a $70-\mathrm{kg}$ person, and from 40 to $120 \mathrm{ng} / \mathrm{ml}$ in a person of low $(50 \mathrm{~kg})$ body weight. These findings argue for the consideration of age- and weight-based dose adjustments in older people. Previously conducted clinical studies have shown an increase in $\mathrm{CL}$ and extended $t^{1} / 2$ in patients with renal impairment (Rosenzweig et al. 2002). As a result, a $50 \%$ reduction in daily dose is advised in moderate renal disease (GFR 30-60 $\mathrm{ml} / \mathrm{min}$ ) and a $66 \%$ reduction in severe renal disease (GFR is $10-30 \mathrm{ml} / \mathrm{min}$ ). The PK model developed in this analysis included eight $A D$ patients with moderate renal disease, a common finding in older patients, and one advantage of building the model from a representative clinical dataset. Our findings suggest that, for patients aged 65 years and above who do not have severe renal impairment, age and weight could be considered in place of renal status, to guide dose reductions.

The published literature on amisulpride (Mauri et al. 2014; Sparshatt et al. 2009) has consistently reported higher dose-corrected concentrations in women during TDM, and it has been assumed that gender differences in drug clearance contribute to this (Coukell and
Benfield 1996). However, data from the four large, naturalistic studies which have contributed most to our understanding of amisulpride PK are comprised predominantly of patients below 65 years and include minimal data on patients aged 80 years and above (Bergemann et al. 2004; Muller et al. 2009, 2007, 2006). In the current study, there were no gender differences in dose-corrected concentrations at steady state, and neither was there an impact of gender on the variability in CL. Our findings suggest that, after the age of 65 years, no additional dose adjustments are required on the basis of gender, after accounting for age and body weight.

There are several limitations to the analysis, including the relatively small sample size and sparse sampling in the clinical study. This meant that it was not possible to estimate within-subject (inter-occasion) variability in CL, which reduced the predictive power of the model and contributed to the high residual unexplained variability $(53 \%)$. It was beyond the scope of the current analysis to examine the contribution of other covariates such as concomitant medication, as the study was not powered to incorporate multiple categorical variables into the covariate model. This is however balanced by the fact that amisulpride is not a CYP450 substrate, which reduces the likelihood of potential drug interactions, and patients who were being prescribed drugs known to interact with amisulpride, including OCT substrates lithium and clozapine (Bowskill et al. 2012), were excluded from participation in the study. We cannot completely rule out the possibility that changes in renal function 
Table 3 Pharmacokinetic model estimates for group 1 and the combined (groups 1 and 2) sample

\begin{tabular}{|c|c|c|c|c|}
\hline \multirow[t]{2}{*}{ Parameter (units) } & \multicolumn{2}{|l|}{ Group $1(n=20)$} & \multicolumn{2}{|l|}{ Combined $(n=45)$} \\
\hline & Estimate & RSE (\%) & Estimate & RSE (\%) \\
\hline $\mathrm{ka}$ & 0.87 & 14 & 0.85 & 16 \\
\hline $\mathrm{Cl}$ & 84 & 7 & 54.3 & 8 \\
\hline$\beta-{ }_{-C l, w e i g h t}$ & 0.75 & ne & 0.75 & ne \\
\hline$\beta-{ }^{-} \mathrm{Cl}$,Age & ne & ne & $-2.9\left(p=2.3^{\mathrm{e}-007}\right)$ & \\
\hline V1 & $\begin{array}{l}668 \text { (Men) } \\
399 \text { (Women) }\end{array}$ & $\begin{array}{l}15 \\
13\end{array}$ & 455 & 13 \\
\hline$\beta$-v1, weight & 1 & ne & ne & ne \\
\hline$\beta$-v1,Gender & $-0.52\left(p=6.6^{\mathrm{e}-005}\right)$ & 25 & ne & ne \\
\hline Q & 117 & 15 & 111 & 16 \\
\hline$\beta-_{\mathrm{Q}, \text { weight }}$ & 0.75 & ne & ne & ne \\
\hline V2 population & 808 & 12 & 736 & 11 \\
\hline$\beta-\mathrm{v} 2$,weight & 1 & ne & ne & ne \\
\hline \multicolumn{5}{|l|}{ Random effect } \\
\hline$\omega_{-} \mathrm{ka} \%$ & 37 & 21 & 48 & 24 \\
\hline$\omega_{-} \mathrm{Cl} \%$ & 31 & 16 & 36 & 16 \\
\hline$\omega_{-} \mathrm{V} 1 \%$ & 42 & 21 & 43 & 27 \\
\hline$\omega_{-} \mathrm{Q} \%$ & 61 & 19 & 63 & 20 \\
\hline$\omega_{-} \mathrm{V} 2 \%$ & 50 & 17 & 46 & 18 \\
\hline$\omega \_\mathrm{Cl} \_\mathrm{V} 1 \%$ & 73 & 19 & 73 & 23 \\
\hline$\omega \_C l \_Q \%$ & 68 & 21 & 60 & 29 \\
\hline$\omega \_V 1 \_Q \%$ & 51 & 48 & 73 & 23 \\
\hline$\omega \_\mathrm{Cl}$-V2\% & 60 & 25 & 66 & 22 \\
\hline$\omega \_V 1 \_V 2 \%$ & 97 & 9 & 90 & 16 \\
\hline$\omega \_$Q_V2\% & 54 & 33 & 60 & 30 \\
\hline \multicolumn{5}{|l|}{ Residual error } \\
\hline$\sigma($ group 1$) \%$ & 13 & 5 & 13 & 6 \\
\hline$\sigma($ group 2$) \%$ & - & - & 53 & 24 \\
\hline
\end{tabular}

$k a$ absorption constant, $\mathrm{Cl}$ apparent clearance from central compartment, $V 1$ central volume of distribution, $Q$ intercompartmental clearance, $V 2$ peripheral volume of distribution, $\omega$ inter-individual variability (expressed as a percentage), $\sigma$ residual unexplained variability (expressed as a percentage and separated on the basis of group), weight $\log$ transformed and centred around a standard $70 \mathrm{~kg}$ weight, age log transformed and centred around the mean, RSE relative standard error, ne not estimated through the course of treatment contributed to the observed variability in $\mathrm{CL}$, as additional monitoring of renal function was not routinely carried out during dose titration, and there was wide variability in length of follow-up due to technical difficulties around the imaging procedure. Compliance is another important source of potential variability in blood concentration and particularly relevant in cognitively impaired patients. As a result, compliance was carefully monitored (caregiver report and tablet counts) and facilitated by the clinical team.

Despite the limitations of the AD dataset, it represents the first PK data on amisulpride within the context of therapeutic, steady state dosing in a clinical population who reached the extreme end of the age spectrum. The magnitude of the change indicates that therapeutic blood concentrations are achieved at lower doses and that age and body weight could be considered in place of renal status to guide dose adjustments. Obtaining additional steady state data on elderly people will help to further refine the model predictions regarding gender, concomitant medications and co-morbid health conditions, including pre-existing renal impairment.

Previous studies which have used a population approach to explore PK profiles of psychotropic drugs in older AD patients have produced mixed findings. For example, PK models developed using data from the Clinical Antipsychotic Trials of Intervention Effectiveness (CATIE) trials for Alzheimer's disease (AD) and schizophrenia (SZ) have shown a significant effect of age on clearance of the active metabolite $(9-\mathrm{OH}$ risperidone) of risperidone (Feng et al. 2008), whereas interindividual variability in olanzapine clearance was accounted for by factors other than age (gender, smoking and African- 
Fig. 2 Visual predictive checks (VPC): $95 \%$ prediction intervals around the 5th, 50th and 95th percentiles are shown for the final model after analysis of group 1 data alone, overlaid to group 1 observed 5th, 50th and 95th percentiles (a), and after analysis of the combined dataset overlaid to group 1 observed 5th, 50th and 95th percentiles (b), and overlaid to group 2 observed 5th, 50th and 95th percentiles (c)
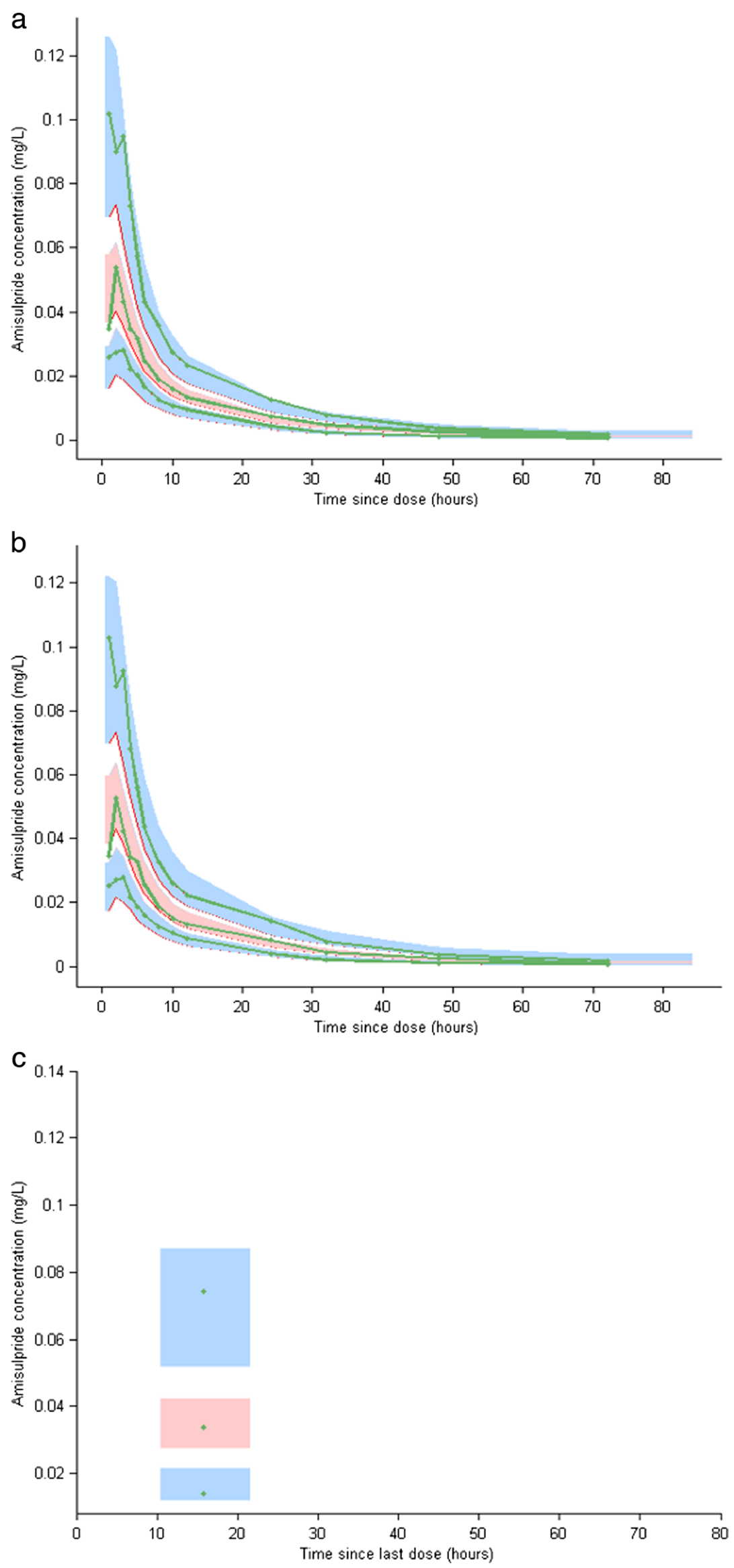
Fig. 3 Simulated amisulpride concentrations (converted to $\mathrm{ng} /$ $\mathrm{ml})$ at $15 \mathrm{~h}$ post-dose in a population of 100 people in each of the following categories: 65,75 or 85 years old; and average $(70 \mathrm{~kg})$ or low $(50 \mathrm{~kg})$ body weight, across the prescribed dose range $(25,50,75 \mathrm{mg}$ daily). Observed data from the current study are represented as circles, binned by age $(65 \pm 5,75 \pm 5$, $85 \pm 5$ years $)$, weight $(50 \pm 10$, $70 \pm 10 \mathrm{~kg})$ and dose

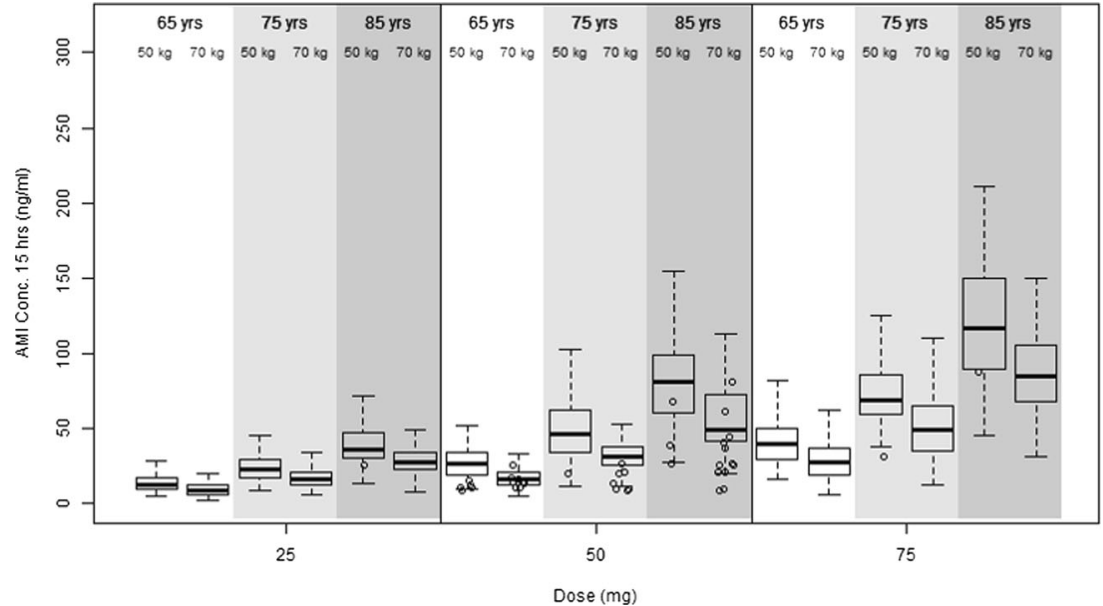

American race) (Bigos et al. 2008b). These data, and a recent publication from the Citalopram in Alzheimer's disease (CitAD) study, which showed significant and clinically relevant effects of age and gender on metabolic clearance of Rbut not S-citalopram (Akil et al. 2016), serve to emphasise the importance of extending pharmacological modelling to representative older clinical populations, to meaningfully refine and optimise age- and disease-specific dose adjustments.

The very low doses of amisulpride prescribed to $\mathrm{AD}$ patients in this study are considerably lower than those used to treat psychotic symptoms in schizophrenia (Hiemke et al. 2011; Sparshatt et al. 2009) and at the lower end of the suggested efficacious dose range from open label studies which have similarly prescribed amisulpride off licence to treat patients with $\mathrm{AD}$ and psychotic symptoms (50-200 mg) (Lim et al. 2006; Mauri et al. 2006). This reflects the primary aim of the study, which was to establish the minimum clinically effective dose required to reduce symptoms without EPS. As a result, dose titration regimens commenced at doses as low as $25 \mathrm{mg}$ (half a tablet) and increased to an optimum, based on response and side effect profile. Of the 25 patients included in the study, only one achieved a blood concentration (109 $\mathrm{ng} / \mathrm{ml})$ within the therapeutic range recommended for the treatment of positive symptoms in schizophrenia (Hiemke et al. 2011; Sparshatt et al. 2009). This suggests that either patient were sub-optimally treated in the current study or that the target therapeutic range is lower in older $\mathrm{AD}$ patients, as a result of age- and/or disease-related changes in central pharmacokinetics (Clark-Papasavas et al. 2014; Seeman 2014) or altered pharmacodynamics (neurotransmitter, receptor or signal transduction level), which lower the therapeutic D2/3 occupancy range (Graff-Guerrero et al. 2015; Uchida et al. 2009a). This will be investigated in future analyses, which will aim to combine the PK model with data on $\mathrm{D} 2 / 3$ receptor imaging and clinical outcome, to explore PK occupancy profiles, establish the target concentration and D2/3 occupancy range to avoid non-response and EPS, and further inform $\mathrm{AD}$-specific dose adjustments.

Acknowledgments The project and salary of SR were funded by the National Institute for Health Research (NIHR) (PCDKBRA) during the course of the study. RH is also supported by the NIHR UCLH BRC. We would like to acknowledge participants, carers and clinical teams in Mental Health for Older Adult services in the South London and Maudsley NHS Foundation Trust who made this possible, the research team, the CRN network who facilitated recruitment and the London Pharmacometric Interest Group (University College London) for their invaluable advice. Demographic and pharmacokinetic data obtained in 20 elderly healthy volunteers were provided by SANOFI-AVENTIS R\&D, from the report entitled "Amisulpride: study of pharmacokinetics and safety following a single oral administration (50 mg tablets) in the elderly" (SYNTHELABO report no. 95-00596-EN-00). These data were supplied by SANOFI-AVENTIS R\&D in the interest of supporting medical research in very elderly patients. Sanofi does not support any use of the medicine outside the locally approved labelling.

\section{Compliance with ethical standards}

Conflict of interest The authors report no conflicts of interest.

Open Access This article is distributed under the terms of the Creative Commons Attribution 4.0 International License (http:// creativecommons.org/licenses/by/4.0/), which permits unrestricted use, distribution, and reproduction in any medium, provided you give appropriate credit to the original author(s) and the source, provide a link to the Creative Commons license, and indicate if changes were made.

\section{References}

Akil A et al. (2016) A population pharmacokinetic model for R- and Scitalopram and desmethylcitalopram in Alzheimer's disease patients with agitation. J Pharmacokinet Pharmacodyn 43:99-109. doi:10.1007/s10928-015-9457-6

Bergemann N, Kopitz J, Kress KR, Frick A (2004) Plasma amisulpride levels in schizophrenia or schizoaffective disorder. Eur 
Neuropsychopharmacol J Eur Coll Neuropsychopharmacol 14:245 250. doi:10.1016/j.euroneuro.2003.09.001

Bigos KL, Chew ML, Bies RR (2008a) Pharmacokinetics in geriatric psychiatry. Curr Psychiatry Rep 10:30-36

Bigos KL et al. (2008b) Sex, race, and smoking impact olanzapine exposure. J Clin Pharmacol 48:157-165

Bowskill SV, Patel MX, Handley SA, Flanagan RJ (2012) Plasma amisulpride in relation to prescribed dose, clozapine augmentation, and other factors: data from a therapeutic drug monitoring service, 2002-2010. Humanist Psychol 27:507-513. doi:10.1002/hup.2256

Clark-Papasavas C et al. (2014) Towards a therapeutic window of D2/3 occupancy for treatment of psychosis in Alzheimer's disease, with $[18 \mathrm{~F}]$ fallypride positron emission tomography. Int J Geriatr Psychiatry 29:1001-1009. doi:10.1002/gps.4090

Cockcroft DW, Gault MH (1976) Prediction of creatinine clearance from serum creatinine. Nephron 16:31-41

Coukell ALSC, Benfield P (1996) Amisulpride: a review of its pharmacodynamic and pharmacokinetic properties and therapeutic efficacy in the management of schizophrenia. CNS Drugs 6:237-256

Cummings JL, Mega M, Gray K, Rosenberg-Thompson S, Carusi DA, Gornbein J (1994) The neuropsychiatric inventory: comprehensive assessment of psychopathology in dementia. Neurology 44:23082314

Dos Santos Pereira JN et al. (2014) The poorly membrane permeable antipsychotic drugs amisulpride and sulpiride are substrates of the organic cation transporters from the SLC22 family. AAPS J 16: 1247-1258. doi:10.1208/s12248-014-9649-9

Duffull SB, Wright DF, Winter HR (2011) Interpreting population pharmacokinetic-pharmacodynamic analyses - a clinical viewpoint. Br J Clin Pharmacol 71:807-814. doi:10.1111/j.13652125.2010.03891.x

Dufour A, Desanti C (1988) Pharmacokinetics and metabolism of amisulpride. Ann Psychiatr 3:298-305

Ette EI, Williams PJ, Lane JR (2004) Population pharmacokinetics III: design, analysis, and application of population pharmacokinetic studies. Ann Pharmacother 38:2136-2144. doi:10.1345/aph.1E260

Feng Y et al. (2008) Population pharmacokinetic analysis for risperidone using highly sparse sampling measurements from the CATIE study. Br J Clin Pharmacol 66:629-639. doi:10.1111/j.13652125.2008.03276.x

Folstein MF, Folstein SE, McHugh PR (1975) "mini-mental state". A practical method for grading the cognitive state of patients for the clinician. J Psychiatr Res 12:189-198

Graff-Guerrero A et al. (2015) Evaluation of antipsychotic dose reduction in late-life schizophrenia: a prospective dopamine D2/3 receptor occupancy study. JAMA Psychiatry 72:927-934. doi:10.1001 /jamapsychiatry.2015.0891

Haenisch B, Drescher E, Thiemer L, Xin H, Giros B, Gautron S, Bonisch $H$ (2012) Interaction of antidepressant and antipsychotic drugs with the human organic cation transporters hOCT1, hOCT2 and hOCT3. Naunyn Schmiedeberg's Arch Pharmacol 385:1017-1023. doi:10.1007/s00210-012-0781-8

Hamon-Vilcot B et al. (1998) Safety and pharmacokinetics of a single oral dose of amisulpride in healthy elderly volunteers. Eur J Clin Pharmacol 54:405-409

Hiemke $\mathrm{C}$ et al. (2011) AGNP consensus guidelines for therapeutic drug monitoring in psychiatry: update 2011. Pharmacopsychiatry 44 : 195-235. doi:10.1055/s-0031-1286287

Holford NH (1996) A size standard for pharmacokinetics. Clin Pharmacokinet 30:329-332

Jennum P, Baandrup L, Ibsen R, Kjellberg J (2015) Increased all-cause mortality with use of psychotropic medication in dementia patients and controls: a population-based register study. Eur Neuropsychopharmacol J Eur Coll Neuropsychopharmacol 25: 1906-1913. doi:10.1016/j.euroneuro.2015.08.014
Jeste DV et al. (2008) ACNP white paper: update on use of antipsychotic drugs in elderly persons with dementia. Neuropsychopharmacology 33:957-970

Jonker JW, Schinkel AH (2004) Pharmacological and physiological functions of the polyspecific organic cation transporters: OCT1, 2, and 3 (SLC22A1-3). J Pharmacol Exp Ther 308:2-9. doi:10.1124 /jpet.103.053298

Kuhn E, Lavielle M (2005) Maximum likelihood estimation in non-linear mixed effects models. Comput Stat Data Anal 49:1020-1038

Lako IM, van den Heuvel ER, Knegtering H, Bruggeman R, Taxis K (2013) Estimating dopamine D(2) receptor occupancy for doses of 8 antipsychotics: a meta-analysis. J Clin Psychopharmacol 33:675681. doi:10.1097/JCP.0b013e3182983ffa

Leon C, Gerretsen P, Uchida H, Suzuki T, Rajji T, Mamo DC (2010) Sensitivity to antipsychotic drugs in older adults. Curr Psychiatry Rep 12:28-33. doi:10.1007/s11920-009-0080-3

Lim HK, Pae CU, Lee C, Lee CU (2006) Amisulpride versus risperidone treatment for behavioral and psychological symptoms in patients with dementia of the Alzheimer type: a randomised, open, prospective study. Neuropsychobiology 54:247-251

Lotrich FE, Bies RR, Smith GS, Pollock BG (2006) Relevance of assessing drug concentration exposure in pharmacogenetic and imaging studies. J Psychopharmacol 20:33-40. doi:10.1177 /1359786806066044

Mangoni AA, Jackson SH (2004) Age-related changes in pharmacokinetics and pharmacodynamics: basic principles and practical applications. Br J Clin Pharmacol 57:6-14

Mauri M, Mancioli A, Rebecchi V, Corbetta S, Colombo C, Bono G (2006) Amisulpride in the treatment of behavioural disturbances among patients with moderate to severe Alzheimer's disease. Acta Neurol Scand 114:97-101. doi:10.1111/j.16000404.2006.00660.x

Mauri MC, Paletta S, Maffini M, Colasanti A, Dragogna F, Di Pace C, Altamura AC (2014) Clinical pharmacology of atypical antipsychotics: an update. EXCLI J 13:1163-1191

Maust DT, Kim HM, Seyfried LS, Chiang C, Kavanagh J, Schneider LS, Kales HC (2015) Antipsychotics, other psychotropics, and the risk of death in patients with dementia: number needed to harm. JAMA Psychiatry 72:438-445. doi:10.1001/jamapsychiatry.2014.3018

McKeith IG et al. (1996) Consensus guidelines for the clinical and pathologic diagnosis of dementia with Lewy bodies (DLB): report of the consortium on DLB international workshop. Neurology 47:11131124

McKhann G, Drachman D, Folstein M, Katzman R, Price D, Stadlan EM (1984) Clinical diagnosis of Alzheimer's disease: report of the NINCDS-ADRDA work group under the auspices of Department of Health and Human Services Task Force on Alzheimer's disease. Neurology 34:939-944

Merle L, Laroche ML, Dantoine T, Charmes JP (2005) Predicting and preventing adverse drug reactions in the very old. Drugs Aging 22: 375-392

Mould DR et al. (2002) Population pharmacokinetic and adverse event analysis of topotecan in patients with solid tumors. Clin Pharmacol Ther 71:334-348. doi:10.1067/mcp.2002.123553

Muller MJ, Eich FX, Regenbogen B, Sachse J, Hartter S, Hiemke C (2009) Amisulpride doses and plasma levels in different age groups of patients with schizophrenia or schizoaffective disorder. J Psychopharmacol 23:278-286. doi:10.1177 /0269881108089806

Muller MJ, Regenbogen B, Hartter S, Eich FX, Hiemke C (2007) Therapeutic drug monitoring for optimizing amisulpride therapy in patients with schizophrenia. J Psychiatr Res 41:673-679. doi:10.1016/j.jpsychires.2005.10.003 
Muller MJ, Regenbogen B, Sachse J, Eich FX, Hartter S, Hiemke C (2006) Gender aspects in the clinical treatment of schizophrenic inpatients with amisulpride: a therapeutic drug monitoring study. Pharmacopsychiatry 39:41-46. doi:10.1055/s-2006-931540

Rosenzweig P, Canal M, Patat A, Bergougnan L, Zieleniuk I, Bianchetti G (2002) A review of the pharmacokinetics, tolerability and pharmacodynamics of amisulpride in healthy volunteers. Humanist Psychol 17:1-13. doi:10.1002/hup.320

Samson A, Lavielle M (2006) Extension of the SAEM algorithm to leftcensored data in nonlinear nixed-effects model: application to HIV dynamics model. Comput Stat Data Anal 51:1562-1574

Seeman P (2014) Therapeutic occupation of dopamine D2 antipsychotic receptors in Alzheimer's disease. Int J Geriatr Psychiatry 29:1096 doi:10.1002/gps.4133
Simpson GM, Angus JW (1970) A rating scale for extrapyramidal side effects. Acta Psychiatr Scand Suppl 212:11-19

Sparshatt A, Taylor D, Patel MX, Kapur S (2009) Amisulpride - dose, plasma concentration, occupancy and response: implications for therapeutic drug monitoring. Acta Psychiatr Scand 120:416-428. doi:10.1111/j.1600-0447.2009.01429.x

Uchida H, Kapur S, Mulsant BH, Graff-Guerrero A, Pollock BG, Mamo DC (2009a) Sensitivity of older patients to antipsychotic motor side effects: a PET study examining potential mechanisms. Am J Geriatr Psychiatry 17:255-263

Uchida H, Mamo DC, Mulsant BH, Pollock BG, Kapur S (2009b) Increased antipsychotic sensitivity in elderly patients: evidence and mechanisms. J Clin Psychiatry 70:397-405 